\title{
Laser Remote Sensing from ISS: CATS Cloud and Aerosol Level 2 Data Products (Heritage Edition)
}

\author{
Sharon Rodier ${ }^{1,3 *}$, Steve Palm ${ }^{2,4}$, Mark Vaughan ${ }^{3}$, John Yorks',4, Matt McGill ${ }^{4}$, Mike \\ Jensen $^{1,3}$, Tim Murray ${ }^{1,3}$, Chip Trepte ${ }^{3}$ \\ ${ }^{1}$ Science Systems and Applications Inc., Hampton, VA USA *Email: sharon.d.rodier@nasa.gov \\ ${ }^{2}$ Science Systems and Applications Inc., Greenbelt, MD, USA \\ ${ }^{3}$ NASA, Langley Research Center, Hampton, VA, USA \\ ${ }^{4}$ NASA, Goddard Space Flight Center, Greenbelt, MD USA
}

\begin{abstract}
With the recent launch of the Cloud-Aerosol Transport System (CATS) we have the opportunity to acquire a continuous record of spacebased lidar measurements spanning from the Cloud-Aerosol Lidar and Infrared Pathfinder Satellite Observations (CALIPSO) era to the start of the EarthCARE mission. Utilizing existing well-validated science algorithms from the CALIPSO mission, we will ingest the CATS data stream and deliver high-quality lidar data sets to the user community at the earliest possible opportunity. In this paper we present an overview of procedures necessary to generate CALIPSOlike lidar level 2 data products from the CATS level 1 data products.
\end{abstract}

\section{INTRODUCTION}

The Cloud-Aerosol Transport System (CATS) instrument was developed at NASA's Goddard Space Flight Center (GSFC) and deployed to the International Space Station (ISS) on 10 January 2015. CATS is mounted on the Japanese Experiment Module's Exposed Facility (JEM_EF) and will provide near-continuous, altitude-resolved measurements of clouds and aerosols in the Earth's atmosphere. The CATS ISS orbit path provides a unique opportunity to capture the full diurnal cycle of cloud and aerosol development and transport, allowing for studies that are not possible with the lidar aboard the CALIPSO platform, which flies in the sun-synchronous ATrain orbit. The CATS instrument consists of two high repetition rate lasers operating at two wavelengths (1064 nm and $532 \mathrm{~nm}$ ) with four configurable fields of view (FOV). By combining a specific laser and FOV(s) the CATS instrument can be configured for three primary operational modes (see [1] for details). In science mode 1, laser 1 measures elastic backscatter and volume depolarization in a dual FOV mode (left FOV and right FOV) at $1064 \mathrm{~nm}$ and $532 \mathrm{~nm}$. In science mode 2, laser 2 measures elastic backscatter and volume depolarization at $1064 \mathrm{~nm}$ and makes High Spectral Resolution Lidar (HSRL) measurements at $532 \mathrm{~nm}$ via the forward FOV. The final configuration is science mode 3 , which uses laser 2 to measure elastic backscatter and volume depolarization at 532 and $1064 \mathrm{~nm}$ via the aft FOV.

One of the primary science objectives of CATS is to continue the CALIPSO aerosol and cloud profile data record to provide continuity of lidar climate observations during the transition from CALIPSO to EarthCARE. To accomplish this, the CATS project at NASA's Goddard Space Flight Center (GSFC) and the CALIPSO project at NASA's Langley Research Center (LaRC) are closely collaborating to develop and deliver a full suite of CALIPSO-like level 2 data products that will be produced using the newly acquired CATS level 1B data whenever CATS is operating in science modes 1 or 3 . The CALIPSO mission is now well into its ninth year of on-orbit operations, and has developed a robust set of mature and well-validated science algorithms to retrieve the spatial and optical properties of clouds and aerosols from multi-wavelength lidar backscatter signals [2]. By leveraging both new and existing NASA technical resources, this joint effort by the CATS and CALIPSO teams will deliver validated lidar data sets to the user community at the earliest possible opportunity. The science community will have access to two sets of CATS Level 2 data products. The "Operational" data products will be produced by the GSFC CATS 
team utilizing the new instrument capabilities (e.g., multiple FOVs and $1064 \mathrm{~nm}$ depolarization), while the "Heritage" data products created using the existing CALIPSO algorithms and the CATS $532 \mathrm{~nm}$ channels and the total $1064 \mathrm{~nm}$ channel.

Throughout this abstract we outline the development of the CATS Level 2 Heritage (L2H) software and data products and describe the modifications made to the ingested CATS Level 1B data stream and the CALIPSO Level 2 processing algorithms in order to successfully interface two disparate data processing systems. Simulated CATS Level 1B data provided an initial assessment of CATS Heritage Level 2 data quality.

\section{METHODOLOGY}

Producing high quality CALIPSO-like Level 2 products requires addressing differences between the instrument sampling rates, vertical resolution, and signal-to-noise (SNR) characteristics. To ingest the CATS data, the $532 \mathrm{~nm}$ and $1064 \mathrm{~nm}$ attenuated backscatter channels must be converted from single shot profiles sampled at $\sim 350 \mathrm{~m}$ horizontally with a uniform $60 \mathrm{~m}$ vertical resolution spanning a total of $30 \mathrm{~km}(28 \mathrm{~km}$ to $-2 \mathrm{~km})$, to the CALIPSO $5 \mathrm{~km}$ downlinked major frame structure that consists of 15 profiles sampled at $\sim 330$ m horizontally that span $42 \mathrm{~km}$ (40 km to -2 $\mathrm{km})$ vertically and are averaged onboard both vertically and horizontally to different spatial resolutions that vary with range from the lidar. Additional required parameters such as molecular and ozone number density are also calculated and ancillary instrument parameters are extracted to create required CALIPSO parameters such as QC Flags, noise scale factors, and RMS background measurements. A set of four software modules (called Product Generation Executables or PGEs) were constructed to accomplish these tasks:

1) Ingest/Archive: receives CATS data files from GSFC and archives them for ordering by the public via the LaRC Atmospheric Sciences Data Center.

2) L1B Pre-Processor: converts CATS L1B into CALIPSO-like L1B by applying CALIPSO's onboard averaging scheme to the CATS data.

3) L2 FOV Merge: while each CATS FOV is processed separately by the Heritage software, the results from both FOVs are reported in single L2H data files. This is consistent with the CATS L1B files, which also report all FOVs in a single file for each orbit segment.

4) CATS Lidar Browse Image creation: generates images of the CATS L1B and L2H data products for display on the CATS web pages.

Figure 1 illustrates the Heritage processing flow for Science Mode 1 from ingest to pre-processing, execution of the L2 algorithms, to post-processing and finally archival of the CALIPSO-like data sets. Science Mode 3 follows a similar flow, but does not require the FOV Merge PGE since CATS data is acquired using only a single FOV.

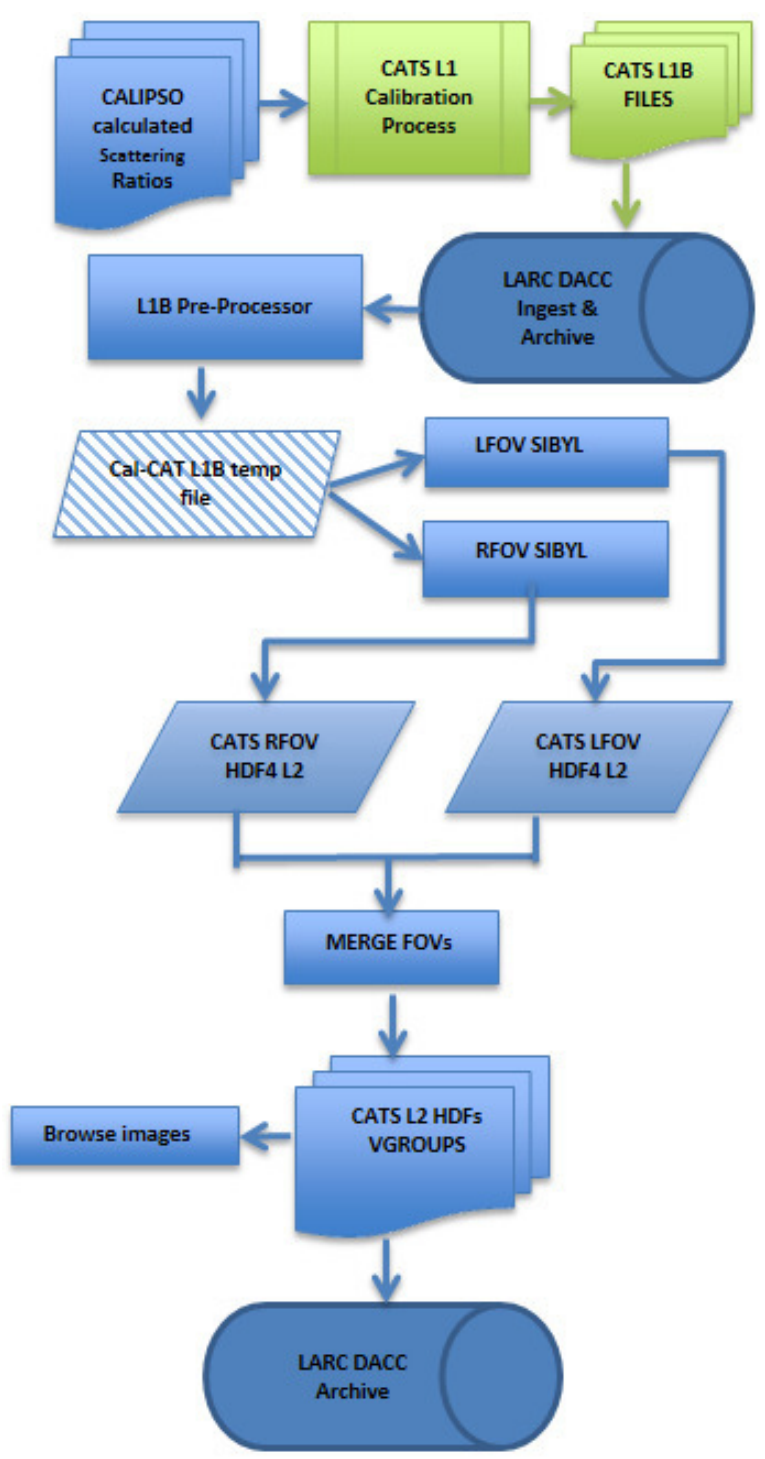

Figure 1: CATS Heritage Processing Flow for Science Mode 1 
Due to SNR differences between the two instruments, turning of the detection levels threshold will be required to accurately perform feature classification of the clouds and aerosols in each scene. Preliminary results from the first CATS downlinks show that the CATS SNR is higher than the CALIPSO SNR at night, especially at $1064 \mathrm{~nm}$. However, the CATS daytime SNR is lower than CALIPSO and, for reasons not yet fully understood, somewhat lower than prelaunch predictions. With respect to the Level 2 Heritage products, these differences are expected to have minimal impact on the quality of the layer detection and optical properties results reported in the $\mathrm{L} 2 \mathrm{H}$ products, largely due to the extensive spatial averaging done by the CALIPSO retrieval algorithms.

\section{RESULTS}

The development and validation of the Heritage data products benefited substantially from high quality CATS L1B simulation data. Multiple orbits created from the meteorological products generated by NASA's Global Modeling and Assimilation Office (GMAO) provided realistic scenes of clouds and aerosol at varying heights and optical depths throughout the atmosphere. These simulations provided the baseline examples of day-night SNR differences that were used to determine the prelaunch configuration (e.g., layer detection thresholds) of the CATS Heritage retrieval system. Figures $2 \mathrm{a}, 2 \mathrm{~b}$ and $2 \mathrm{c}$ show an extended cirrus cloud deck above boundary layer aerosols during a continuous transition from daytime (left side) to nighttime (right side) data acquisition. While the differences in SNR are plainly evident (Figures 2a and 2b), the retrieval algorithms are uniformly successful in properly locating and identifying the layers, irrespective of lighting conditions (Figure 2c).

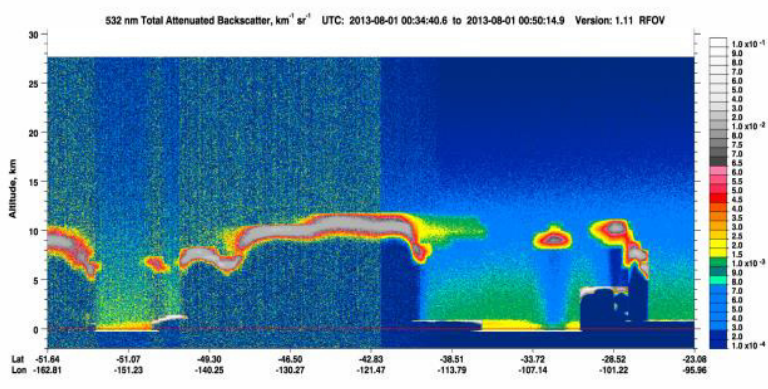

Figure 2a: Simulated CATS L1B $532 \mathrm{~nm}$ total attenuated backscatter showing cirrus clouds above boundary layer aerosols.

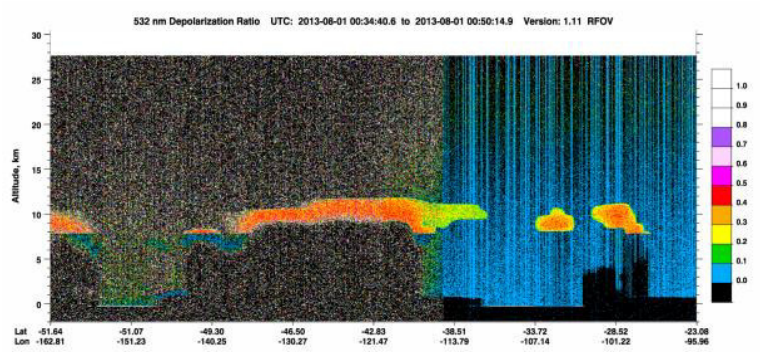

Figure 2b: CATS L1B $532 \mathrm{~nm}$ volume depolarization ratios

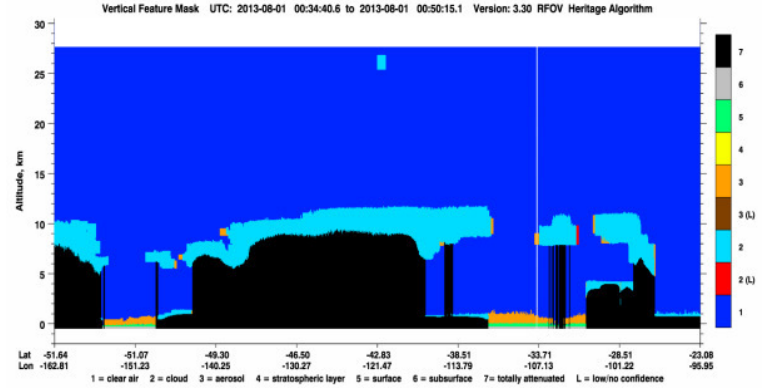

Figure 2c: CATS L2H vertical feature mask showing locations of cloud (light blue) and aerosol (orange) layers identified in the CATS. The high altitude $(\sim 26 \mathrm{~km})$ layer at $\sim 43^{\circ} \mathrm{S}$ is a false positive introduced by high levels of solar background noise.

The CATS Lidar Browse Image PGE will produce all the standard L1 and L2 images generated by the CALIPSO analyses. In additional, some CATS specific images such as plots of the $1064 \mathrm{~nm}$ volume depolarization will also be produced. Figures 3a-3e show a subset of the CATS L1 and L2 browse products that will be available to the science community.

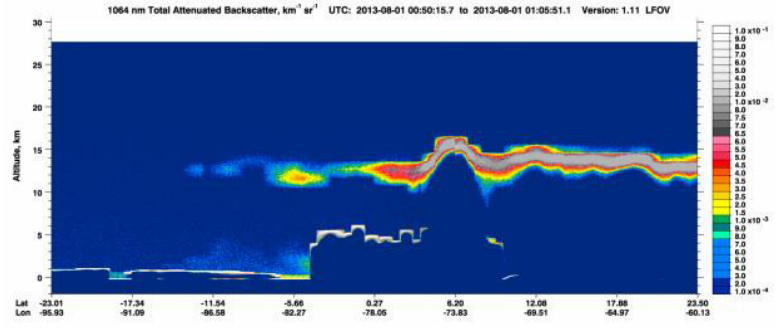

Figure 3a: CATS L1B Simulated $1064 \mathrm{~nm}$ total attenuated backscatter showing cirrus and highaltitude aerosol lying over (left) a stratus deck embedded in a height-varying aerosol layer and (center) midlevel opaque water clouds. 


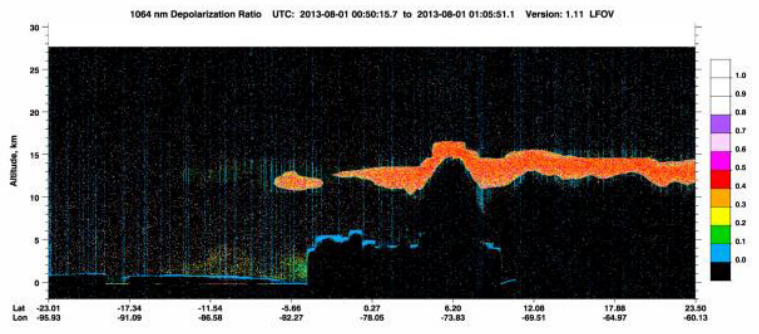

Figure 3b: CATS L1B 1064 nm volume depolarization ratios

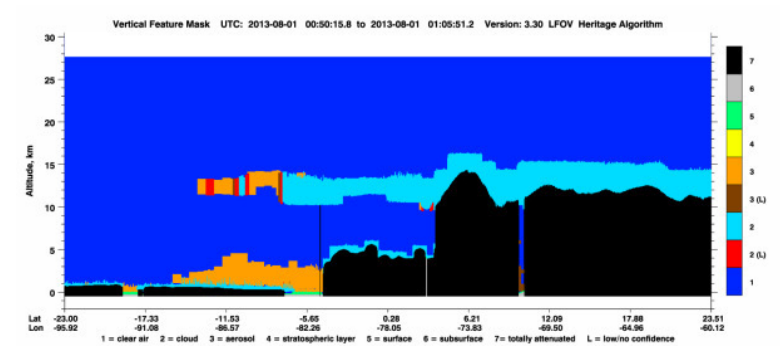

Figure 3c: CATS L2H vertical feature mask for the data shown in Figures $3 a$ and $3 c$

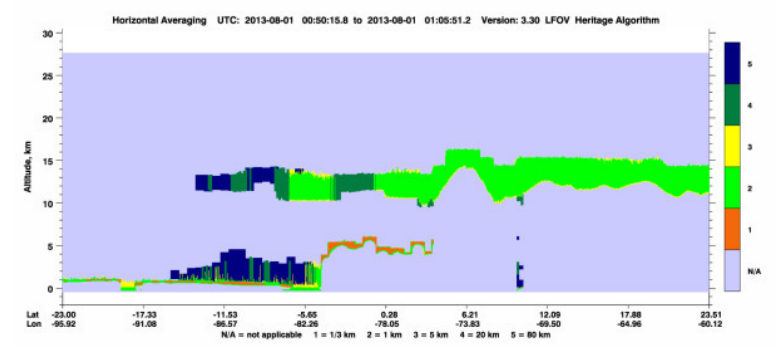

Figure 3d: CATS L2H horizontal averaging required for layer detection

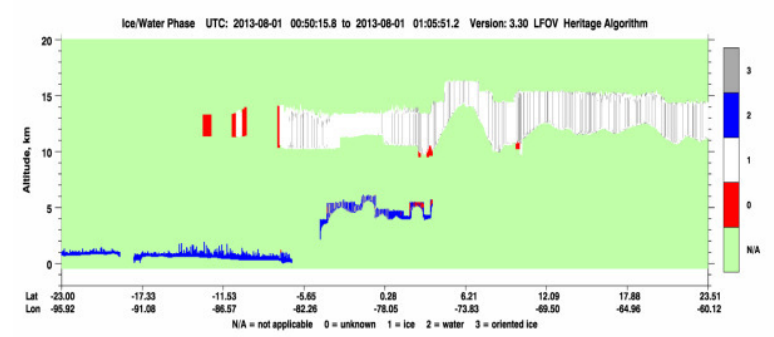

Figure 3e: CATS L2H ice-water phase

\section{CONCLUSIONS}

The first CATS data was telemetered back to Earth on 10 February 2015, and as of this writing the GSFC CATS team is completing their initial post-launch verifications of their data handling, geolocation and instrument calibration algorithms and code. Once these quality assessment procedures have been successfully completed, routine transfer of the CATS L1B files from GSFC to LaRC will begin.

The CATS L2H data products shown in the images above were generated with a wholly unmodified version of the CALIPSO Level 2 Version 3.30 analysis software. While this software is quite capable of handing the CATS simulated data, some minor modifications may be required to best accommodate the real world data stream. Furthermore, a certain amount of configuration and quality assurance testing remains to be done to optimize $\mathrm{L} 2 \mathrm{H}$ retrieval performance for the actual (as opposed to simulated) CATS data. Upon the conclusion of these activities, production of the CATS L2H data products will begin. At present, the LaRC CATS team is anticipating public release of the $\mathrm{L} 2 \mathrm{H}$ products by early-to-mid summer of 2015.

\section{REFERENCES}

[1] Yorks, J. E., M. J. McGill, S. P. Palm, D. L. Hlavka, P. A. Selmer, E. P. Nowottnick, M. A. Vaughan and S. D. Rodier, 2015: An Overview of the Cloud-Aerosol Transport System (CATS) Processing Algorithms and Data Products, Proceedings of the $27^{\text {th }}$ International Laser Radar Conference (ILRC 27)

[2] Winker, D. M., M. A. Vaughan, A. H. Omar, Y. Hu, K. A. Powell, Z. Liu, W. H. Hunt, and S. A. Young, 2009: "Overview of the CALIPSO Mission and CALIOP Data Processing Algorithms", J. Atmos. Oceanic Technol., 26, 2310-2323, doi:10.1175/2009JTECHA1281.1. 